\title{
OBSERVACIONES SOBRE LA FERTILIDAD DIFERENCIAL DE DOS MORFOTIPOS ALARES EN PEREGRINUS MAIDIS (ASHMEAD) (HEMIPTERA: DELPHACIDAE) EN CONDICIONES DE LABORATORIO
}

\author{
OBSERVATIONS ABOUT DIFFERENTIAL FERTILITY OF TWO ALAR \\ MORPHOTYPES ON PEREGRINUS MAIDIS (ASHMEAD) (HEMIPTERA: \\ DELPHACIDAE) UNDER LABORATORY CONDITIONS
}

Tommy C. Rioja ${ }^{1}$; Héctor Vargas ${ }^{1}$; Dante Bobadilla ${ }^{1}$

\section{RESUMEN}

Bajo las condiciones agroecológicas de los valles de Arica, Región de Arica y Parinacota, Chile, el delfácido del maíz Peregrinus maidis (Ashmead) se comporta como estricto monófago, colonizando cultivos de maíz amiláceo, Zea mays L. var. Amylacea, y maíz dulce, Z. mays L. var. Saccharata. Ambos tipos de maíz se cultivan durante todo el año para consumo como hortaliza (choclo), y su colonización por P. maidis se efectúa con eficacia gracias a la existencia de dos morfotipos alares, i.e. ejemplares macrópteros encargados de la dispersión e individuos braquípteros que cumplen el rol de hacer crecer rápidamente la población local. Observaciones de campo, complementadas con mediciones realizadas en microjaulas, y también sobre ejemplares aislados en laboratorio, permitieron establecer que las hembras macrópteras presentaron un período promedio de preoviposición mayor (17,8 $\pm 1,2$ días) que las hembras braquípteras ( $12 \pm 1,1$ días). En cambio, estas últimas mostraron un período promedio de oviposición más largo ( $20,7 \pm 6,7$ días) que las hembras macrópteras ( $8,7 \pm 1,5$ días). Asimismo, las hembras braquípteras depositaron en promedio un mayor número de huevos $(157,8)$ que las hembras macrópteras $(49,7)$. Analizados estos datos mediante la prueba t de Student, se encontró que para los tres parámetros anteriormente indicados las diferencias estadísticas entre ambos morfotipos alares son muy significativas $(P \leq 0,001)$. Se concluye que estas diferencias son congruentes con los roles distintos y complementarios que cumplen ambos morfotipos en la dispersión, colonización y explotación del recurso alimentario.

Palabras clave: Planthopper, Peregrinus maidis, Zea mays L., macrópteros, braquípteros, oviposición.

\begin{abstract}
Under agroecological conditions of the Arica valleys, Region of Arica y Parinacota, Chile, the planthopper or corn delphacid, Peregrinus maidis (Ashmead), behavior as strict monophagous, colonizing amylaceous cornfields, Zea mays L. var. Amylacea, and sweet corn, Z. mays $L$. var. Saccharata. Both corn types are cultivated during all year to consume fresh product (ear of corn), and P. maidis colonization are achieved with the existence of two alar morphotypes, i. e. macropterous forms to colonization, and brachypterous forms in charges to make up increase the local population. Field station observations, with measurements done in microcages, and laboratory specimen, allowed establish that female macropterous forms presented a larger preoviposition (17.8 \pm 1.2 days) that brachypterous forms ( $12 \pm 1.1$ days). Besides, its last showed a larger oviposition period ( $20.7 \pm 6.7$ days) that macropterous females $(8.7 \pm 1.5$ days). Also, the brachypterous females deposited on average a bigger number of eggs (157.8) than those macropterous females (49.7). This records were analyzed through the t of Student test; it found that for three parameters previously indicated the estadistical differences between both alar morphotypes are very significant $(P \leq 0.001)$. These differences have relation with each morphotype roll in the dispersion, colonization and exploitation of alimentary resource.
\end{abstract}

Key words: Planthopper, Peregrinus maidis, Zea mays L., macropterous, brachypterous, oviposition.

\section{INTRODUCCIÓN}

La familia Delphacidae incluye aproximadamente 2.000 especies, la mayoría de las cuales se encuentran en todos los biomas de la tierra incluyendo la tundra ártica, desiertos y bosques tropicales. Estos artrópodos se alimentan de la savia elaborada, ingiriendo nutrientes del floema de la planta. La mayoría de los

1 Laboratorio de Entomología, Facultad de Ciencias Agronómicas, Universidad de Tarapacá, Campus Azapa, Arica - Chile. 
delfácidos tienen un rango muy limitado de plantas hospedantes, con un $70 \%$ de ellos comportándose como monófagos. Muchas especies pertenecientes a esta familia se alimentan de monocotiledóneas especialmente plantas de las familias Juncaceae, Cyperaceae y Poaceae, aunque ciertos delfácidos tienen afinidad hacia algunas dicotiledóneas como la familia Asteraceae. Con respecto a la oviposición de los delfácidos, las hembras insertan sus huevos dentro del tejido vegetal usando su ovipositor parecido a una sierra (Wilson, 1997).

La mayoría de los delfácidos exhiben dimorfismo alar (Zera \& Denno, 1997). Los adultos braquípteros tienen alas reducidas, por lo que no pueden volar, mientras que los adultos macrópteros poseen alas completamente desarrolladas, los que pueden recorrer grandes distancias (Langelotto \& Denno, 2001). Las hembras braquípteras son generalmente más fértiles y con un período de preoviposición más corto que las macrópteras (Denno \& Roderick, 1990). En relación a la condición de polimorfismo alar de Peregrinus maidis, Fernández-Badillo \& Clavijo (1990) señalan que esta condición no estaría determinada por un solo factor, sino que se encuentra regida por la acción cuantitativa de varios factores. Así, bajas densidades poblacionales favorecen la aparición de una mayor proporción de individuos braquípteros, mientras que altas densidades pueden determinar una total aparición de individuos macrópteros (Langelotto \& Denno, 2001; Brentassi \& Marino de Remes Lenicov, 2007). La "calidad del alimento" parece también tener un efecto sobre el polimorfismo alar del insecto (Zera, 2004).

Las hormonas juveniles son una clase de reguladores sesquiterpenoides, que controlan la metamorfosis en insectos inmaduros y la reproducción en insectos adultos. Estas hormonas son sintetizadas y secretadas por las corpora allata, par de glándulas endocrinas con terminaciones nerviosas al cerebro (Li et al., 2003). La actividad de las Corpora allata es dependiente y modulada por factores internos y ambientales tales como estado de desarrollo, fotoperíodo, diapausa, alimentación, estado nutricional y apareamiento (Li et al., 2003). Chapman (1978) menciona que en áfidos la aparición de individuos ápteros (sin alas) es resultado de un exceso de la hormona juvenil; así, el balance hormonal puede ser influido por una gran variedad de factores externos. Asimismo, Romoser \& Stoffolano (1998) indican que los factores ambientales que tienen influencia en el polimorfismo alar, en diferentes especies, son por ejemplo: el fotoperíodo, la temperatura, cambios en la planta-huésped (cambios nutritivos), además Zera (2004) señala que tanto factores ambientales anteriormente señalados como factores genéticos influyen en el polimorfismo alar.

En Chile, el delfácido del maíz Peregrinus maidis se encuentra presente en los valles costeros de Azapa y Lluta, afectando con intensidad variable los cultivos de maíz amiláceo, Zea mays L. var. Amylacea, y maíz dulce, Z. mays L. var. Saccharata. Bajo las condiciones agroecológicas de estos valles, este delfácido se comporta como estricto monófago, colonizando estos tipos de maíz que se cultivan durante todo el año para consumo como hortaliza. La colonización y crecimiento poblacional del fitófago se efectúan con eficacia gracias a la existencia de dos morfotipos alares; ejemplares macrópteros encargados de la dispersión e individuos braquípteros que cumplen el rol de hacer crecer rápidamente la población local (Rioja, 2003; Rioja et al., 2006).

\section{MATERIAL Y MÉTODOS}

El estudio se llevó a cabo bajo condiciones de laboratorio en el Campo Experimental de la Facultad de Ciencias Agronómicas de la Universidad de Tarapacá, ubicado en el km 12 del valle de Azapa, Región de Arica y Parinacota.

En 13 placas de Petri se introdujo un trozo de

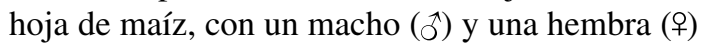
de P. maidis (individuos de edad conocida obtenidos en laboratorio); así, al producirse la oviposición dicho trozo de hoja era extraído y reemplazado por otro diariamente para cada hembra ( $\approx$ repetición). Se registró el tiempo que transcurrió entre la última muda hasta la primera postura (período de preoviposición), con observaciones diarias y a la misma hora. El conteo de huevos se realizó en el laboratorio, haciendo disecciones de los trozos de hojas bajo estereomicroscopio utilizando un bisturí. Las temperaturas al interior del laboratorio se registraron mediante un termómetro de máxima y de mínima; como también la humedad relativa registrada a través de un higrómetro. Así, las temperaturas al interior del laboratorio para el período de preoviposición fue de $17,8 \pm 4^{\circ} \mathrm{C}$ y para el período de oviposición $18,9 \pm 4{ }^{\circ} \mathrm{C}$. La humedad relativa al interior del laboratorio fue de $65 \pm 5 \%$. Posteriormente se aplicó un ANOVA y el test 
T-Student para verificar las diferencias significativas entre las hembras macrópteras y braquípteras para los períodos de preoviposición, oviposición y fecundidad promedio según morfotipo alar.

\section{RESULTADOS Y DISCUSIÓN}

La cópula se lleva a cabo en horas de la mañana y tarde (Figura 1), demorando alrededor de 40-60 minutos en completarse. Bajo las condiciones del estudio, el morfotipo alar aparentemente no influyó en el comportamiento reproductivo de la especie, observándose cópula entre individuos del mismo morfotipo y también entre machos macrópteros y hembras braquípteras o viceversa. Con respecto a esto, Denno \& Roderick (1990) y Langelotto \& Denno (2001) indican que, en los delfácidos, ambos sexos producen señales acústicas mediante vibraciones con sus abdómenes, dorsoventralmente, sin tocar la epidermis del tejido vegetal; dichas vibraciones son transferidas a la planta hospedante, a través de sus patas y tal vez con sus estiletes; estas señales acústicas sirven para: gatillar conductas de agresión, localización y atracción entre sexos, reconocimiento de la pareja. Vibraciones similares fueron observadas en laboratorio en hembras de $P$. maidis, corroborando así lo señalado por los autores. Por otra parte, Olvido et al. (2003) señalan que el macropterismo en grillos podría tener un costo considerable en el fitness o adecuación biológica de machos macrópteros, los cuales son menos exitosos que sus contrapartes de alas cortas en la atracción de hembras para realizar la cópula. Además, el macropterismo en Nilaparvata lugens (Hemiptera: Delphacidae) está asociado a largos tiempos de desarrollo huevo-adulto, resultando en bajo éxito de copulación de los machos de esta especie.

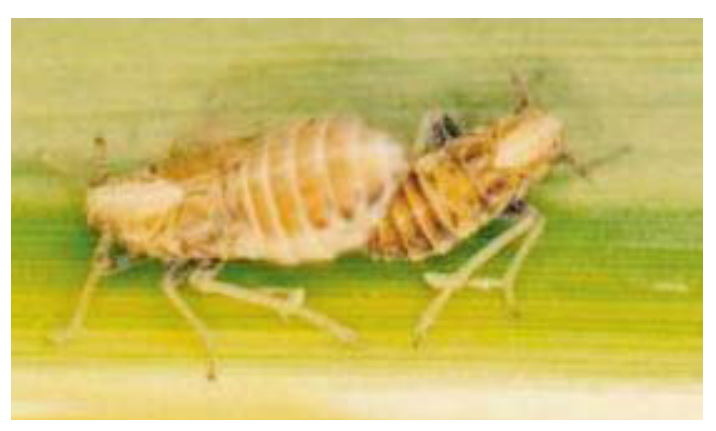

Figura 1. Cópula entre individuos braquípteros de $P$. maidis, en condiciones de laboratorio.
Peregrinus maidis inserta sus huevos en el interior del tejido vegetal mediante su ovipositor en forma de sierra, los cuales quedan depositados oblicuamente, para luego cubrirlos con una sustancia blanco-serosa que tras endurecerse deja manchas blancas en la epidermis del tejido vegetal; así, deposita sus huevos en zonas de tejido tierno, como son la nervadura central de las hojas y sus cercanías, tanto en el haz como envés de las mismas; en el interior de vainas foliares, en las brácteas que envuelven la mazorca, y en las brácteas que envuelven la inflorescencia masculina (panoja) (Rioja et al., 2006). Este mecanismo de inserción evitaría la deshidratación de los huevos del delfácido del maíz, principalmente distribuido sobre cultivos de maíz y sorgo en zonas tropicales y subtropicales del mundo.

De acuerdo a las observaciones realizadas en laboratorio, se comprobó que las hembras macrópteras de esta especie (Figura 2) presentaron un período de preoviposición mayor ( $\overline{\mathrm{x}}=17,8 \pm 1,2 \mathrm{ds}$.) que las braquípteras (Figura 3 ) ( $\bar{x}=12 \pm 1,1$ ds.). En cambio,

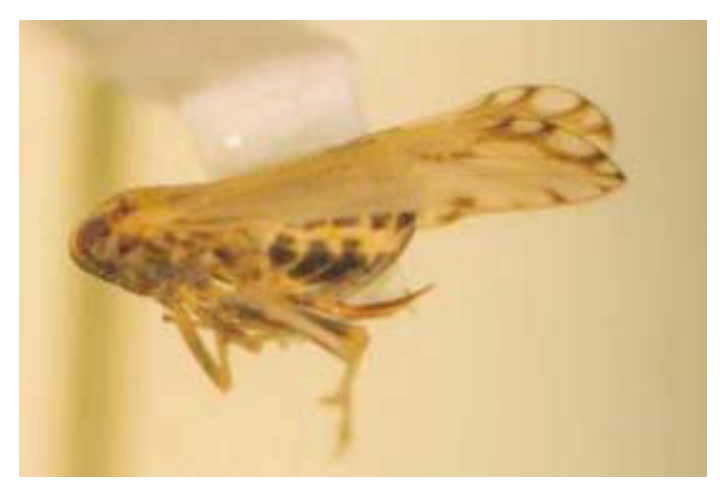

Figura 2. Hembra macróptera de $P$. maidis. Vista lateral. Barra $=0,8 \mathrm{~mm}$.

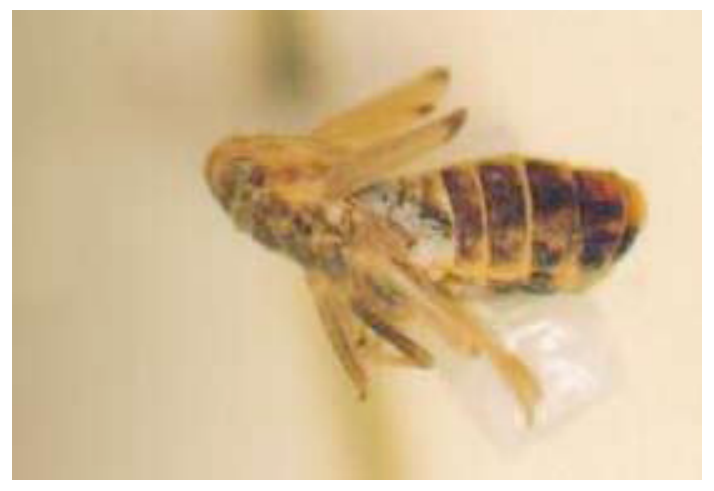

Figura 3. Hembra braquíptera de $P$. maidis. Vista lateral. Barra $=0,66 \mathrm{~mm}$. 
estas últimas mostraron un período de oviposición más largo $(\bar{x}=20,7 \pm 6,7$ ds. $)$ que las hembras macrópteras ( $\bar{x}=8,7 \pm 1,5$ ds.). Asimismo, las hembras braquípteras depositaron un mayor número de huevos ( $\bar{x}=157,8 \pm 40,9)$ que las macrópteras $(\bar{x}=49,7 \pm 25)$. Analizados estos datos mediante la prueba $t$ de Student, se encontró que para los tres parámetros anteriormente indicados las diferencias estadísticas entre ambos morfotipos alares son muy significativas $(\mathrm{P} \leq 0,01)$ (Figuras 4, 5 y 6$)$.
Por lo tanto, el período de oviposición y el número total de huevos ovipuestos fue mayor en las hembras braquípteras; mientras que el período de preoviposición fue mayor para las macrópteras, diferencias que coinciden con la función que cumple cada morfotipo alar dentro de la especie. Las macrópteras tienen la función de colonizar i.e. largos períodos de preoviposición, seguidos de cortos períodos de oviposición, favorecen la dispersión de la especie; mientras que las braquípteras

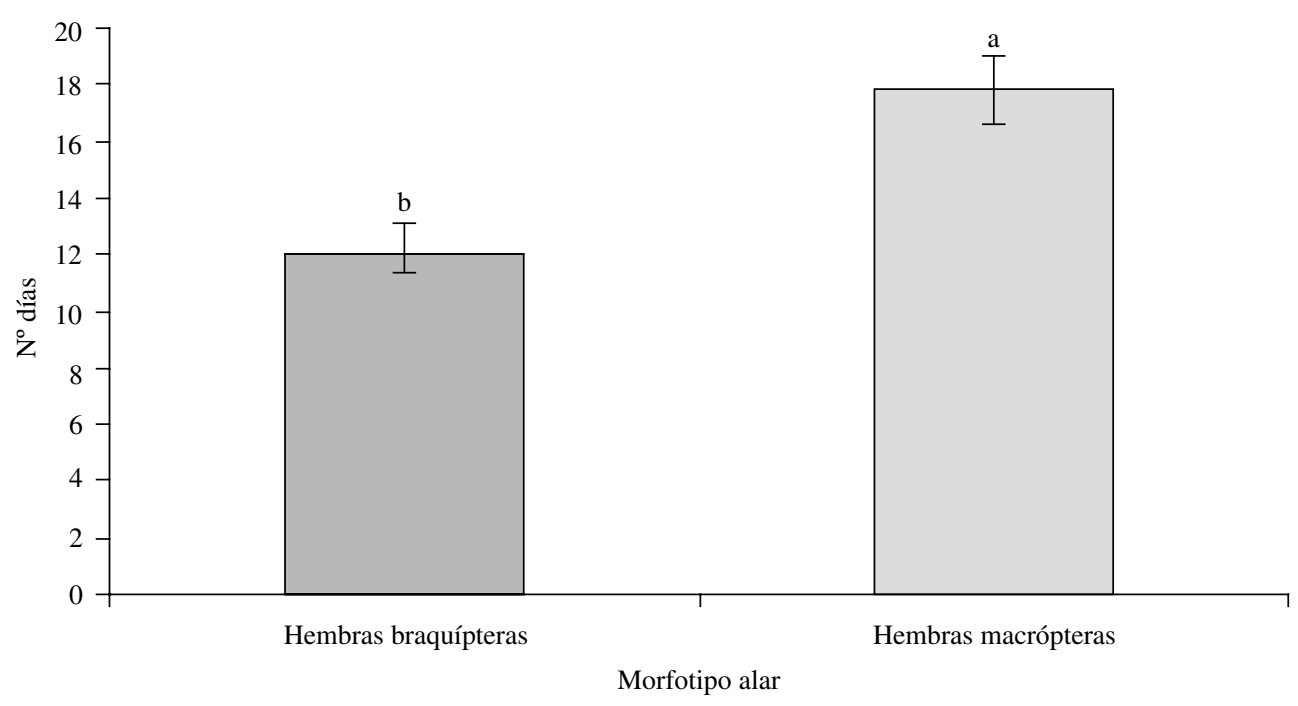

Figura 4. Duración del período de preoviposición de P. maidis, en condiciones de laboratorio, según el morfotipo alar (en días).

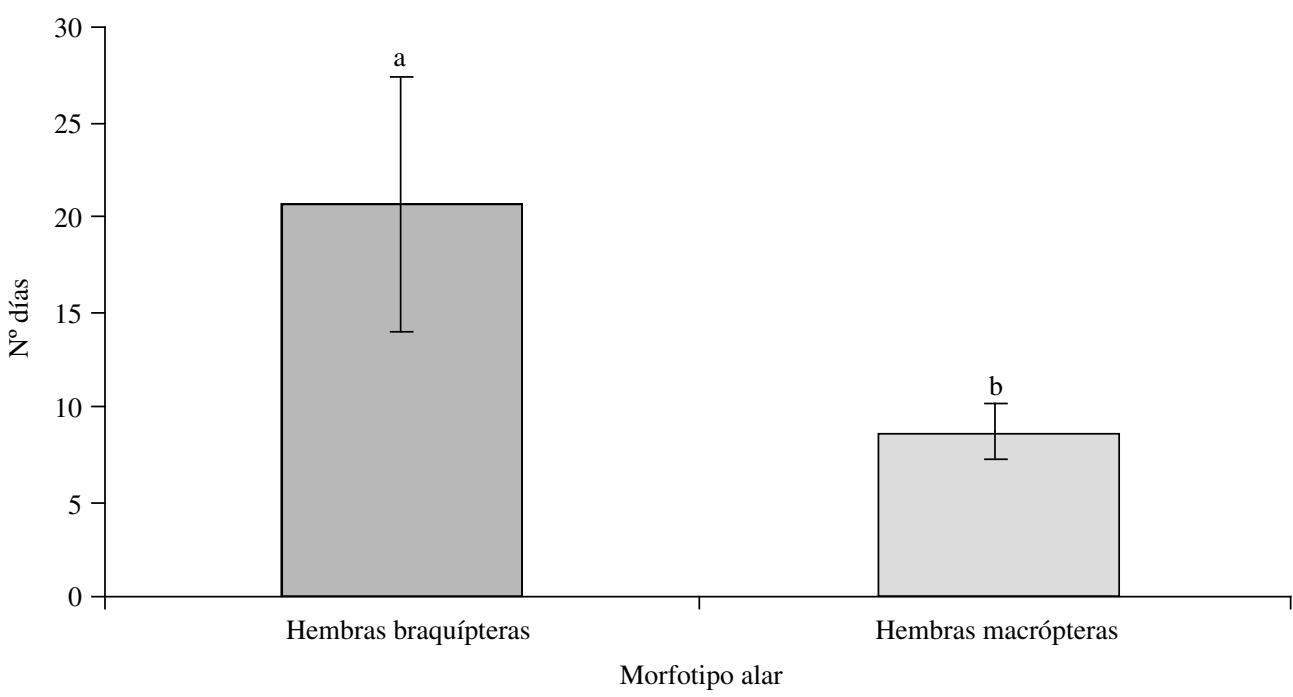

Figura 5. Duración del período de oviposición de P. maidis, en condiciones de laboratorio, según el morfotipo alar (en días). 


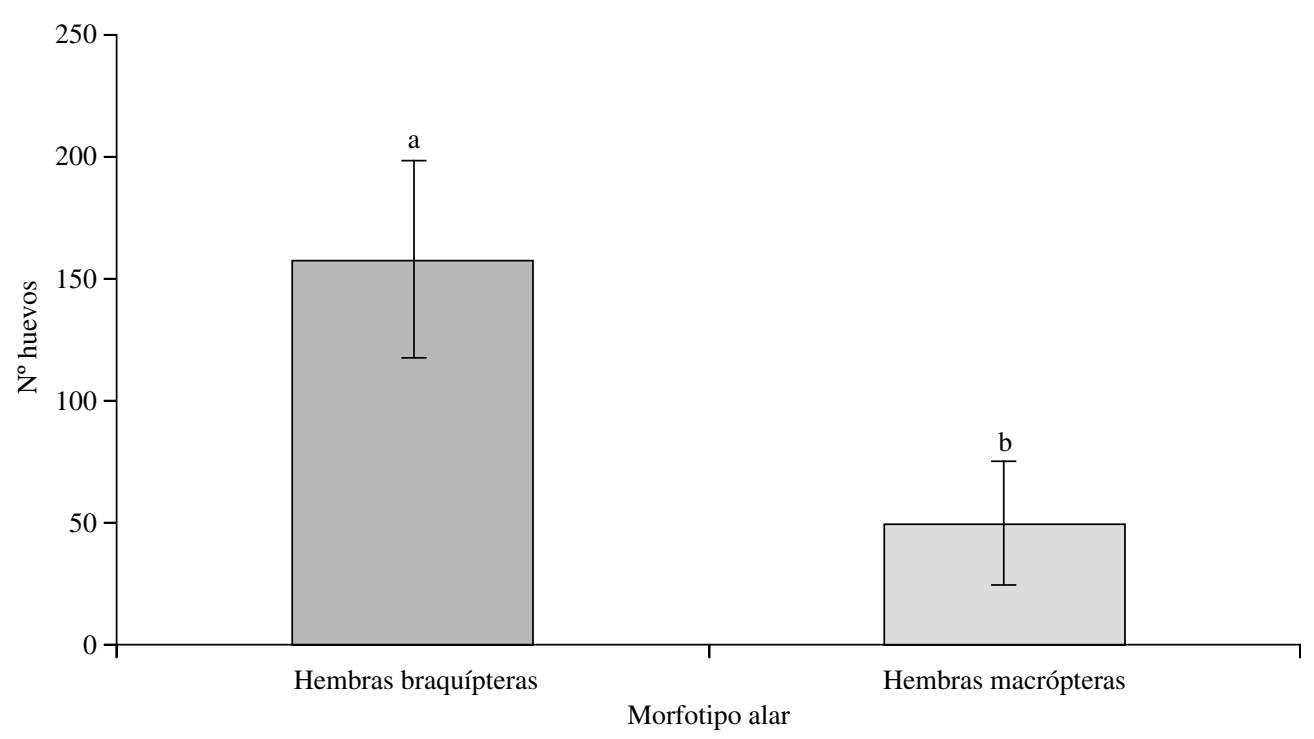

Figura 6. Número promedio de huevos ovipuestos por hembras de P. maidis en condiciones de laboratorio, según el morfotipo alar.

tienen la función reproductiva i.e. cortos períodos de preoviposición, seguidos de largos períodos de oviposición, favorecen un rápido crecimiento poblacional del delfácido, de este modo se optimiza el uso del recurso alimentario y, cuando este se agota, se reinicia el proceso de emigración para colonizar, posteriormente, nuevos cultivos de maíz.

La calidad del alimento sería uno de los factores determinantes para la aparición del macropterismo en esta especie; se debe agregar que este factor puede tener varios elementos dependientes, ya que las altas temperaturas provocan un aumento de la población debido a cortos períodos de desarrollo en la etapa embrionaria y ninfal para llegar más rápido a la edad reproductiva (huevo-adulto), con la consiguiente escasez del alimento, provocando la aparición de individuos de alas completamente desarrolladas. Dicha escasez de alimento no implica necesariamente una disminución nutricional para el insecto, influyendo solamente en la cantidad de alimento disponible para una población excesivamente grande del delfácido. Braendle et al. (2006) señalan que, en áfidos, las condiciones ambientales inducen a los individuos alados y no alados, además el incremento en la densidad de los áfidos desencadena la formación de alas en muchas especies.

P. maidis, a nivel mundial, es plaga reconocida del maíz y el sorgo (Sorghum vulgare L.) (Wilson, 2005), lo cual indicaría una coevolución del delfácido del maíz con dichos cereales. Esto, por supuesto, relacionado con la fisiología de ambas gramíneas. En varios estudios realizados en 12 diferentes especies de áfidos no se pudo confirmar la hipótesis de que una nutrición pobre es responsable para un incremento en individuos alados. En muchos estudios recientes se comprobó el efecto que tiene la planta-huésped en la producción de individuos alados, lo cual se debe al excesivo contacto físico entre los áfidos; sin embargo, una deficiente calidad nutricional de la planta puede desencadenar la producción de alas en algunas especies (Braendle et al., 2006). Así, el dimorfismo alar de $P$. maidis estaría relacionado estrechamente con la fenología y fisiología del cultivo del maíz amiláceo en los valles de Lluta y Azapa, ya que existiría una respuesta a nivel fisiológico, traspasada desde la planta-huésped al insecto, que desencadenaría en el fitófago cambios hormonales en los últimos estadios ninfales, teniendo como consecuencia una redistribución de la energía, afectando directamente su adecuación biológica, desarrollando por ejemplo musculatura de vuelo en desmedro de la fecundidad del macróptero, ya que su función principal es la supervivencia de la especie mediante la búsqueda de nuevos cultivos de maíz, por comportarse en la biocenosis de los valles de Lluta y Azapa como estricto monófago (Rioja et al., 2006)).

En relación a lo anteriormente señalado, Zera (2005) indica que, en grillos alados y no alados, el consumo y la asimilación de nutrientes es similar en cantidad, además las diferencias en relación a la energía localizada entre los morfotipos macrópteros 
vs. braquípteros podrían deberse casi exclusivamente a las diferencias morfoespecíficas en localizaciones internas de los nutrientes (i.e., una compensación interna) más que las diferencias morfoespecíficas en adquisición de los nutrientes. Así, los grillos con capacidad de vuelo destinan gran cantidad de su energía para el crecimiento y mantenimiento de sus desarrollados músculos de vuelo como también para la producción de grandes cantidades de triglicéridos para el funcionamiento de sus alas, lo que, a su vez, inhibiría el crecimiento de los ovarios; aunque no está claro aún si el reducido crecimiento de los ovarios se debe a los nutrientes o al espacio insuficiente para contener grandes ovarios, junto a músculos torácicos bien desarrollados y grandes reservas de lípidos necesarias para la actividad de vuelo. Por lo tanto, los individuos desprovistos de alas, y que poseen mayor fecundidad, tienen una ventaja sustancial de su adecuación biológica (fitness) frente a sus pares alados, pero a su vez los morfotipos alados tienen la capacidad de encontrar así nuevos recursos alimenticios y emigrar por el deterioro de la planta huésped.

Con respecto a la influencia que tendrían los cambios de los niveles hormonales en los delfácidos, Bertuso \& Tojo (2002) encontraron en el delfácido Nilaparvata lugens, la hormona juvenil (HJ) III, tanto en ninfas como en adultos. Ambos autores sugieren que altos niveles de la HJ III, presente en los instares ninfales tres y cuatro, podrían determinar el fenotipo braquíptero de dicho delfácido. La $\mathrm{HJ}$ regularía el crecimiento de los oocitos y la maduración de los ovarios, siendo más rápida en hembras braquípteras que en hembras macrópteras, por poseer las primeras niveles más altos de HJ. Los diferentes niveles de la HJ, entre las dos formas alares, concuerdan con el síndrome migratorio influido por la $\mathrm{HJ}$, y la evidente diferencia en la capacidad de vuelo $v s$. la compensación de la fecundidad, observado en especies con dimorfismo sexual.

\section{LITERATURA CITADA}

BERTUSO, A.; TOJO, S. 2002. The nature and titer of juvenile hormone in the brown planthhopper, Nilaparvata lugens (Homoptera: Delphacidae) in relation to wing morphogenesis and oocyte development. Applied Entomology Zoology. 37 (1): 117-125.

BRAENDLE, C.; DAVIS, G.; BRISSON, J.; STERN, D. 2006. Wings dimorphism in aphids. Heredity. 97: 192-199.

BRENTASSI, M.; MARINO DE REMES LENICOV, A. 2007. Feeding behavior of the vector Delphacodes kuscheli

\section{CONCLUSIONES}

1. El delfácido del maíz Peregrinus maidis presenta dos morfotipos alares en la biocenosis del valle de Azapa.

2. El macropterismo de $P$. maidis tiene la función de dispersión de la especie, y este rol se ve facilitado por largos períodos de preoviposición seguidos de cortos períodos de oviposición.

3. El braquipterismo de $P$. maidis tiene la función de incrementar la población local del delfácido, la cual se favorece con cortos períodos de preoviposición seguidos de largos períodos de oviposición.

4. Las hembras braquípteras son más fecundas que las hembras macrópteras de $P$. maidis, produciendo una mayor cantidad de huevos que sus pares de alas desarrolladas.

5. El dimorfismo alar de esta especie se encuentra estrechamente relacionado con la fenología del cultivo del maíz amiláceo en los valles de Lluta y Azapa.

6. $\quad$ El morfotipo alar de $P$. maidis responde a factores ambientales e internos de la planta del maíz, los cuales influyen a nivel genético y fisiológico del delfácido, redestinando el espacio torácico de esta especie a favor de la producción de grandes músculos de vuelo en desmedro de la fecundidad (etapa de emigración) o la formación de grandes ovarios (etapa de colonización) según sea la señal bioquímica.

7. Se recomienda realizar estudios a nivel de fisiología del insecto para verificar los niveles de hormona juvenil presente en ambos morfotipos alares, ya que los niveles de esta hormona influirían en el proceso de metamorfosis y reproducción en los últimos estadios inmaduros de los delfácidos, al igual como ocurre en otros insectos.

(Hemiptera: Fulgoromorpha: Delphacidae) on maize and oat. Ann. Soc. Entomol. Fr. (n.s.) 43 (2): 205-212.

CHAPMAN, R.F. 1978. The Insects: Structure and Function. Great Britain, London. 819 pp.

DENNO, R.; RODERICK, G. 1990. Population Biology of Planthoppers. Annual Review Entomology. 35: 489520.

FERNÁNDEZ-BADILLO,A.; CLAVIJO,S. 1990. Polimorfismo alar de la chicharrita del maíz, Peregrinus maidis (Homoptera: 
Delphacidae) en Venezuela. Revista de la Facultad de Agronomía (Maracay), 16 (1): 27-34.

LANGELOTTO, G.; DENNO, R. 2001. Benefits of dispersal in patchy environments: Mate location by males of a wingdimorphic insect. Ecology 82 (7): 1870-1878.

LI, Y.; HERNÁNDEZ-MARTÍNEZ, S.; UNNITHAN, G.; FEYEREISEN, R.; NORIEGA, F. 2003. Activity of the corpora allata of adult female Aedes aegypti: effects of mating and feeding. Insect Biochemistry and Molecular Biology. 33: 1307-1315.

LI, Y.; KUWANO, E.; NORIEGA, F. 2003. 1,5-Disubstituted imidazoles inhibit juvenile hormona biosynthesis by the corpora allata of the mosquito Aedes aegypti. Journal of Insect Physiology. 49: 1005-1011.

OLVIDO,A.; ELVINGTON,E.; MOUSSEAU,T. 2003. Relative effects of climate and crowding on wings polymorphism in the southern ground cricket, Allonemobius socius (Orthoptera: Gryllidae). Florida Entomologist. 86(2): 158-164.

RIOJA, T. 2003. Biología y Enemigos Naturales de Peregrinus maidis (Ashmead) (Hemiptera: Delphacidae) en el valle de Azapa. Tesis de grado, Facultad de Agronomía, Universidad de Tarapacá, Arica, Chile. 82 p.

RIOJA, T.; VARGAS, H.; BOBADILLA, H. 2006. Biología y enemigos naturales de Peregrinus maidis (Ashmead)
(Hemiptera: Delphacidae) en el valle de Azapa. IDESIA (Chile). 24 (1): 41-48 p.

ROMOSER, W.; STOFFOLANO, J. 1998. The science of entomology. $4^{\mathrm{a}}$ edición, United States. $605 \mathrm{pp}$.

WILSON, S. 1997. Delphacid Planthoppers (Homoptera: Fulgoroidea: Delphacidae) of the Yukon. In: Insects of the Yukon. Biological Survey of Canada (Terrestrial Arthropods), Ottawa. Danks, H. V. \& Downes J. A. (eds.). 377-385 pp.

WILSON, S. 2005. Keys to the families of fulgoromorpha with emphasis on planthoppers of potential economic importance in the Southeastern United States (Hemiptera: Auchenorrhyncha). Florida Entomologist 88 (4): 464481.

ZERA, A. 2004. The endocrine regulation of wing polymorphism in insects: State of the art, recent surprises, and Future Directions. Integr. Comp. Biol. 43: 607-616.

ZERA, A. 2005. Intermediary metabolism and life history tradeoffs: lipid metabolism in lines of the wing-polymorphic cricket, Gryllus firmus, selected for flight capability vs. early age reproduction. Integr. Comp. Biol. 45: 511-524.

ZERA, A. \& R. DENNO. 1997. Physiology and ecology of dispersal polymorphism in insects. Annual Review of Entomology 42: 207-230. 
